\title{
SUFFICIENT CONDITIONS FOR AN OPERATOR-VALUED FEYNMAN-KAC FORMULA
}

\author{
BY \\ MICHAEL DALE GRADY
}

\begin{abstract}
Let $E$ be a locally compact, second countable Hausdorff space and let $X(t)$ be a Markov process with state space $E$. Sufficient conditions are given for the existence of a solution to the initial value problem, $\partial u / \partial t=A u$ $+V(x) \cdot u, u(0)=f$, where $A$ is the infinitesimal generator of the process $X$ on a certain Banach space and for each $x \in E, V(x)$ is the infinitesimal generator of a $C_{0}$ contraction semigroup on another Banach space.
\end{abstract}

1. Introduction. The classical Feynman-Kac formula is given by the function space integral

$$
u(x, t)=E_{x}\left[\exp \left(\int_{0}^{t} V(x(s)) d s\right) f(x(t))\right]
$$

where $x(t)$ is a Markov process with a locally compact, second countable, Hausdorff state space $E, V$ is a bounded, measurable function on $E$ and $E_{x}$ is the expectation with respect to the initial distribution $P_{x}$. This function space integral is a solution to the initial value problem

$$
\partial u / \partial t=A u+V(x) \cdot u, \quad u(0)=f,
$$

where $A$ is the infinitesimal generator of the process $x(t)$.

The operator-valued Feynman-Kac formula is given by a path space integral which gives a solution to (1), where for each $x \in E, V(x)$ is an operator on a linear subspace, $D(V(x))$, of a Banach space, $A$ is the infinitesimal generator of a strongly continuous semigroup of operators on that Banach space and for each $(t, x), u(t, x) \in \mathcal{D}(V(x))$.

In [9] Hersh and Papanicolaou have shown that under some conditions (1) has a solution and they exhibit a solution. They do not, however, set out what the conditions are. In [6] Griego and Hersh show (1) has a solution in the case $E$ is a finite set.

We shall give sufficient conditions on the operators $V(x)$ and on the initial conditions so that a solution to (1) exists.

Presented to the Society, April 12, 1975; received by the editors July 2, 1975.

AMS (MOS) subject classifications (1970). Primary 60J35, 60J45.

Key words and phrases. Function space integrals, semigroups of operators, Markov processes, random evolutions, multiplicative operator functionals. 
The proof will follow that of the classical case given by Dynkin [3]. We shall use the concept of a random evolution introduced in [6] by Griego and Hersh and that of a multiplicative operator functional as in [5].

2. Needed functional analytic results. In this section we will present several results from functional analysis without proof.

Let $E$ be a locally compact, second countable, Hausdorff space, $E$ the Borel $\sigma$-field of subsets of $E$, and $m$ a $\sigma$-finite measure on $(E, E)$. Let $L$ be a separable Banach space.

We shall identify two measurable functions from $E$ to $L$ if they are equal almost everywhere with respect to $m$.

THEOREM 2.1. If $f: E \rightarrow L$ is strongly measurable, then $f$ is Bochner integrable with respect to $m$ if, and only if, $\int_{E}\|f(x)\| d m(x)<\infty$.

The proof of this result can be found in [15, p. 133].

Let us denote by $L^{p}(E, L, m)$ the space of Borel measurable functions $f$ from $E$ to $L$ so that $\int_{E}\|f(x)\|^{p} d m(x)<\infty$. The following result is shown by Zaanen [16, p. 225].

THEOREM 2.2. If $1 \leqslant p<\infty$, then $L^{p}(E, L, m)$ is a Banach space.

The proof of the following can be found in [15, p. 131].

Theorem 2.3 (Pettis' Theorem). $A$ map $f: E \rightarrow L$ is strongly measurable if, and only if, it is weakly measurable and m-almost separably valued.

Proposition 2.4. If $f \in L^{p}(E, L, m)$, then $f$ is strongly measurable.

Proof. By Theorem 2.3, it suffices to show that if $f \in L^{p}$ and $\varphi \in L^{*}$ (the dual of $L$ ), then $\varphi(f)$ is Borel measurable.

But $f \in L^{p}$ implies $f$ is Borel measurable and $\varphi \in L^{*}$ implies $\varphi$ is continuous, hence, $\varphi(f)$ is Borel measurable. This completes the proof.

3. Preliminaries. Let $X=\left(\Omega, \Sigma, \Sigma_{t}, x(t), P_{x}, x \in E\right)$ be a Markov process with locally compact, second countable, Hausdorff state space $E$. Assume that $X$ has continuous paths. Let $P_{x}(x(t) \in A)$ be the transition probabilities of $X$, i.e. $P(t, x, A)=P_{x}(x(t) \in A)$.

We shall suppose as in $\S 2$ that $E$ is the Borel $\sigma$-field on $E$ and that $m$ is a $\sigma$-finite measure on $(E, E)$. Finally, suppose that $L$ is a separable, reflexive Banach space.

Definition 3.1. The measure $m$ is said to be excessive relative to the family $P(t, \cdot \cdot)$ if

$$
m P(t, A) \equiv \int_{E} P(t, x, A) m(d x) \leqslant m(A)
$$

for every $t \geqslant 0$ and $A \in E$. 
Throughout the rest of this paper we shall assume that $m$ is excessive relative to $P(t, ; \cdot)$.

LEMMA 3.2. If $\varphi: E \rightarrow \mathbf{R}$ is measurable, then

$$
\int_{E} E_{x}[\varphi(x(t))] d m(x)=\int_{E} \varphi(x) m P(t, d x) \text { for every } t \geqslant 0 .
$$

Proof. It suffices to show the result when $\varphi$ is a simple function. To that end, let $\varphi=I_{A}$ where $I_{A}$ is the indicator of the set $A \in E$. Then

$$
\begin{aligned}
\int_{E} E_{x}[\varphi(x(t))] d m(x) & =\int_{E} E_{x}\left[I_{A}(x(t))\right] d m(x)=\int_{E} P_{x}(x(t) \in A) d m(x) \\
& =\int_{E} P(t, x, A) d m(x)=m P(t, A)=\int_{E} I_{A}(x) m P(t, d x) .
\end{aligned}
$$

This is the desired result.

Proposition 3.3. If $f \in L^{P}(E, L, m), 1 \leqslant p<\infty$, then for each $t \geqslant 0$ the map $f(x(t)): \Omega \rightarrow L$ is strongly measurable and

$$
\int_{\Omega}\|f(x(t))\| d P_{x}<\infty \text { a.e. } m .
$$

Proof. Since $f$ is Borel measurable and $x(t)$ is $\Sigma$-measurable, we have $f(x(t))$ is Borel measurable, and again by Pettis' Theorem we have $f(x(t))$ is strongly measurable.

Now let $A=\left\{x \in E \mid E_{x}\left(\|f(x(t))\|^{p}\right)=\infty\right\}$, where $E_{x}(Y)$ denotes $\int_{\Omega} Y d P_{x}$ and let $B=\left\{x \in E \mid E_{x}(\|f(x(t))\|)=\infty\right\}$. Since for every $x \in E, P_{x}$ is a probability measure (and hence $L^{p}\left(\Omega, \Sigma, P_{x}\right) \subseteq L^{1}\left(\Omega, \Sigma, P_{x}\right)$ ), we have $B \subseteq A$. We are trying to show that $m(B)=0$ and so it suffices to show $m(A)=0$.

Suppose $m(A)>0$, then

$$
\int_{E} E_{x}\left(\|f(x(t))\|^{p}\right) m(d x)=\infty
$$

But by Lemma 3.2 we have

$$
\int_{E} E_{x}\left(\|f(x(t))\|^{p}\right) m(d x)=\int_{E}\|f(x)\|^{p} m P(t, d x) .
$$

So we have

$$
\begin{aligned}
\infty & =\int_{E} E_{x}\left(\|f(x(t))\|^{p}\right) m(d x)=\int_{E}\|f(x)\|^{p} m P(t, d x) \\
& \leqslant \int\|f(x)\|^{p} m(d x)<\infty .
\end{aligned}
$$

The second to last inequality is due to the fact that $m$ is excessive relative to the family $P(t, ; \cdot)$ and $\|f(x)\|^{p} \geqslant 0$.

The above gives us a contradiction and hence $m(A)=0$, which is the desired result. 
Corollary 3.4. If $f \in L^{P}(E, L, m), 1 \leqslant p<\infty$, then $f(x(t))$ is Bochner integrable with respect to $P_{x}$ for $m$-almost every $x$ in $E$.

Proof. Proposition 3.3 and Theorem 2.1.

We now proceed to define one of the semigroups on $L^{p}(E, L, m)$ which we shall use later.

Definition 3.5. If $f \in L^{p}(E, L, m), 1 \leqslant p<\infty$, then define $(\bar{T}(t) f)(x)=$ $E_{x}(f(x(t)))$, where the integral on the right is a Bochner integral for every $x$ where this makes sense.

Notice that Corollary 3.4 guarantees that this integral makes sense a.e. $m$.

THEOREM 3.6. $\bar{T}(t)$ is a bounded linear operator from $L^{p}(E, L, m)$ to itself.

Proof. First notice that $\bar{T}(t) f: E \rightarrow L$ is defined up to a set of $m$-measure zero, hence, it makes sense to say $\bar{T}(t) f \in L^{p}(E, L, m)$.

In order to show that $\bar{T}(t) f$ is Borel measurable we define

$$
\phi(x)= \begin{cases}E_{x}(f(x(t))), & \text { if } E_{x}\left(\|f(x(t))\|^{p}\right)<\infty, \\ 0, & \text { otherwise. }\end{cases}
$$

Then we need to show that $\phi$ is Borel measurable. Let $\varphi \in L^{*}$. Then

$$
\varphi(\phi(x))= \begin{cases}E_{x}(\varphi(f(x(t)))), & \text { if } E_{x}\left(\|f(x(t))\|^{p}\right)<\infty, \\ 0, & \text { otherwise. }\end{cases}
$$

Now if $\bar{T}(t)$ denotes the semigroup on the integrable Borel measurable functions from $E$ to $\mathrm{R}$ associated with the Markov process $X$, then

$$
\varphi(\phi(x))=\bar{T}(t)(\varphi \circ f)(x) .
$$

Since $\varphi^{\circ} f$ is Borel measurable, we have $\varphi \circ \phi$ is Borel measurable. Hence, $\phi$ is weakly measurable and applying Pettis' Theorem we have $\phi$ is strongly measurable, hence $\phi$ is Borel measurable. But $\phi=\bar{T}(t) f$.

We have already shown in the proof of Proposition 3.3 that

$$
\begin{aligned}
\int_{E}\|\bar{T}(t) f(x)\|^{p} d m(x) & =\int_{E}\left\|E_{x}(f(x(t)))\right\|^{p} d m(x) \leqslant \int_{E}\left\{E_{x}(\|f(x(t))\|)\right\}^{p} d m(x) \\
& \leqslant \int_{E} E_{x}\left(\|f(x(t))\|^{p}\right) d m(x) \quad \text { (by Hölder's Inequality) } \\
& \leqslant \int_{E}\|f(x)\|^{p} d m(x) \quad \text { (Proposition 3.3). }
\end{aligned}
$$

This gives us that $\|\bar{T}(t) f\|_{p} \leqslant\|f\|_{p}$. Hence $\bar{T}(t)$ is bounded. It is clearly linear. This completes the proof.

In order to show that $\bar{T}(t)$ is a semigroup of operators on $L^{p}(E, L, m)$, we first need two lemmas. 
LeMma 3.7. $E_{x}(f(x(t)))=\int_{E} f(y) P(t, x, d y)$, where both integrals are Bochner integrals.

PROOF. This follows from the standard change of variables theorem for Bochner integrals $\left[2\right.$, p. 182] and the fact that $P_{x}\left(x(t)^{-1}(A)\right)=P_{x}(x(t) \in A)=$ $P(t, x, A)$ for all $A \in E, t \geqslant 0$ and $x \in E$.

LEMmA 3.8. If $f \in L^{P}(E, L, m), 1 \leqslant p<\infty$, then

$$
\int_{E} f(y) P(t+s, x, d y)=\int_{E} \int_{E} f(z) P(t, y, d z) P(s, x, d y) .
$$

Proof. It suffices to show the equality for simple functions. To that end, let $f(x)=\Sigma_{i=1}^{n} \alpha_{i} I_{B_{i}}(x)$ where $\alpha_{i} \in L, B_{i} \in E$ and $m\left(B_{i}\right)<\infty$. Then

$$
\begin{array}{r}
\int_{E} f(y) P(t+s, s, d y)=\sum_{i=1}^{n} \alpha_{i} P\left(t+s, x, B_{i}\right)=\sum_{i=1}^{n} \alpha_{i} \int_{E} P\left(t, y, B_{i}\right) P(s, x, d y) \\
=\int_{E} \sum_{i=1}^{n} \alpha_{i} P\left(t, y, B_{i}\right) P(s, x, d y)=\int_{E} \int_{E} f(z) P(t, y, d z) P(s, x, d y) .
\end{array}
$$

This completes the proof.

Before we prove the main result of this section we state a result of Griego [5, Theorem 1.2].

THEOREM 3.9. Let $\{M(t), t \geqslant 0\}$ be a multiplicative operator functional on $(X, L)$. If $L$ is a reflexive Banach space, and if for fixed $\delta>0$,

$$
C \equiv \sup _{0<t<\delta} \sup _{x \in E}\left(E_{x}\left(\|M(t)\|^{q}\right)\right)^{p / q}<\infty
$$

where $1 / p+1 / q=1$ and $1<p<\infty$, then the operators on $L^{p}(E, L, m)$ defined by $S(t) f(x)=E_{x}[M(t) f(x(t))]$ are strongly continuous in $t$.

We now state and prove the main result of this section.

THEOREM 3.10. The family $\{\bar{T}(t), t \geqslant 0\}$ is a strongly continuous contraction semigroup of operators on $L^{p}(E, L, m), 1<p<\infty$.

Proof. The fact that $\bar{T}(t)$ is a contraction map from $L^{p}(E, L, m)$ into itself was established in Theorem 3.6. The strong continuity follows from Theorem 3.9, where our multiplicative operator functional is given by $M(t, \omega)=I$ (the identity operator on $L^{p}(E, L, m)$ ) for all $t$ and $\omega$. Notice that this is the first time the reflexivity of $L$ is used. It remains to show that $\{\bar{T}(t), t \geqslant 0\}$ is a semigroup. We have 


$$
\begin{aligned}
\bar{T}(t+s) f(x) & =E_{x}[f(x(t+s))]=\int_{E} f(y) P(t+s, s, d y) \\
& =\int_{E} \int_{E} f(z) P(t, y, d z) P(s, x, d y) \quad \text { (by Lemma 3.8) } \\
& =\int_{E} \bar{T}(t) f(y) P(s, x, d y) \quad \text { (by Lemma 3.7) } \\
& =\bar{T}(s) \bar{T}(t) f(x) \quad \text { (by Lemma 3.7), }
\end{aligned}
$$

and finally $\bar{T}(0) f(x)=E_{x}[f(x(0))]=f(x)$. Hence, $\{\bar{T}(t), t \geqslant 0\}$ is a semigroup. This completes the proof.

4. The expectation semigroup. In this section we shall build another strongly continuous contraction semigroup on $L^{p}(E, L, m)$ which will be called the expectation semigroup following Griego and Hersh [6].

We need some lemmas.

LEMMA 4.1. If $S_{n}$ and $T_{n}$ are sequences of bounded linear operators on $L$ so that $S_{n} \rightarrow S$ strongly and $T_{n} \rightarrow T$ strongly and if $\left\{\left\|S_{n}\right\|, n \geqslant 1\right\}$ is bounded, then $S_{n} T_{n} \rightarrow S T$ strongly.

The proof is clear so we omit it.

The following result is shown by Khalili $[11$, p. 16].

LEMMA 4.2. If $\left\{f_{n}\right\}$ is a sequence of strongly measurable functions from a measure space to a Banach space, and if $f_{n} \rightarrow f$ pointwise, then $f$ is strongly measurable.

In defining our expectation semigroup we shall use a multiplicative operator functional which will be given by the solution operators to a random evolution equation. We now proceed to set up the desired evolution equations.

Suppose that for each $x \in E$ we have a strongly continuous contraction semigroup of operators $\left\{T_{x}(t), t \geqslant 0\right\}$ on $L$. Denote by $V(x)$ the infinitesimal generator of this semigroup and denote the domain of $V(x)$ by $D(V(x))$.

We shall find it necessary to make the following assumptions which we number for convenience. $t \geqslant 0$.

(A-1) (Commutativity) $T_{x}(s) T_{y}(t)=T_{y}(t) T_{x}(s)$ for every $x, y \in E$ and $s$,

(A-2) $D(V(x))=D$ for all $x \in E$.

(A-3) The map $x \rightarrow V(x) f$ is continuous for every $f \in D$.

I would like to thank the referee for pointing out the following. In the original write-up, I assumed both the hypothesis and the conclusion of the next lemma.

LEMMA 4.3. If the map $x \rightarrow V(x) f$ is continuous for every $f \in D$ then the map $x \rightarrow T_{x}(t) f$ is continuous for every $f \in L$ and $t \geqslant 0$. 
Proof. Let $f \in D$ and suppose that $x_{n} \rightarrow x$. Then we have $T_{x_{n}}(t) f-f$ $=\int_{0}^{t} T_{x_{n}}(s) V\left(x_{n}\right) f d s$ (cf. [3, p. 33]). Writing the same formula with $x$ instead of $x_{n}$, we have

$$
\begin{aligned}
T_{x_{n}}(t) f-T_{x}(t) f= & \int_{0}^{t}\left[T_{x_{n}}(s) V\left(x_{n}\right) f-T_{x}(s) V(x) f\right] d s \\
= & \int_{0}^{t} T_{x_{n}}(s)\left[V\left(x_{n}\right) f-V(x) f\right] d s \\
& +\int_{0}^{t}\left[T_{x_{n}}(s)-T_{x}(s)\right] V(x) f d s .
\end{aligned}
$$

Now let $a_{n}=\left\|V\left(x_{n}\right) f-V(x) f\right\|$. Then we have

$$
\left\|T_{x_{n}}(t) f-T_{x}(t) f\right\| \leqslant t a_{n}+\int_{0}^{t}\left\|\left[T_{x_{n}}(s)-T_{x}(s)\right] V(x) f\right\| d s .
$$

If we let

$$
A_{n}(t)=\max \left\{\left\|T_{x_{n}}(t) f-T_{x}(t) f\right\|,\left\|\left(T_{x_{n}}(t)-T_{x}(t)\right) V(x) f\right\|\right\}
$$

then from the above inequality we see that

$$
A_{n}(t)<t a_{n}+\int_{0}^{t} A_{n}(s) d s
$$

and hence $A_{n}(t) \leqslant a_{n}\left(e^{t}-1\right)$. But now, by hypothesis, $a_{n} \rightarrow 0$ and, hence, $A_{n}(t) \rightarrow 0$ for every $t \geqslant 0$. Therefore, $T_{x_{n}}(t) f \rightarrow T_{x}(t) f$ for all $f \in D$.

Now let $f \in L$ and let $\epsilon>0$. Since $D^{n}$ is dense in $L$, there is a $g \in D$ so that $\|f-g\|<\epsilon$. Now we have

$$
\begin{aligned}
\left\|T_{x_{n}}(t) f-T_{x}(t) g\right\| & \leqslant\left\|T_{x_{n}}(t) f-T_{x_{n}}(t) g\right\|+\left\|T_{x_{n}}(t) g-T_{x}(t) g\right\| \\
& \leqslant\left\|T_{x_{n}}(t)\right\|\|f-g\|+\left\|T_{x_{n}}(t) g-T_{x}(t) g\right\| \\
& \leqslant \epsilon+\left\|T_{x_{n}}(t) g-T_{x}(t) g\right\| .
\end{aligned}
$$

Hence $\operatorname{Lim} \sup _{n}\left\|T_{x_{n}}(t) f-T_{x}(t) g\right\| \leqslant \epsilon$.

Finally we consider the following:

$$
\begin{aligned}
\left\|T_{x_{n}}(t) f-T_{x}(t) f\right\| & \leqslant\left\|T_{x_{n}}(t) f-T_{x}(t) g\right\|+\left\|T_{x}(t) g-T_{x}(t) f\right\| \\
& <\epsilon+\left\|T_{x_{n}}(t) f-T_{x}(t) g\right\|
\end{aligned}
$$

and hence

$$
\underset{n}{\operatorname{Lim} \sup }\left\|T_{x_{n}}(t) f-T_{x}(t) f\right\| \leqslant \epsilon+\underset{n}{\operatorname{Lim} \sup }\left\|T_{x_{n}}(t) f-T_{x}(t) g\right\| \leqslant 2 \epsilon .
$$

Since $\epsilon>0$ was chosen arbitrarily we have $\operatorname{Lim}_{n}\left\|T_{x_{n}}(t) f-T_{x}(t) f\right\|=0$ which is the desired result. 
Goldstein [4] has studied the following initial value problem:

$$
d y / d t=A(t) y, \quad s<t, \quad y(s)=f,
$$

where the initial value $f$ is in some Banach space and for each $t \geqslant s, A(t)$ is the generator of a strongly continuous semigroup of operators on that Banach space. He has shown that under sufficient conditions on the operators $A(t)$ there are solution operators $M(s, t)$ which satisfy the following:

$$
\frac{\partial}{\partial t} M(s, t) f=M(s, t) A(t) f=A(t) M(s, t) f
$$

$$
\frac{\partial}{\partial s} M(s, t) f=-M(s, t) A(s) f=-A(s) M(s, t) f, \quad M(s, s)=I,
$$

where $f$ is an element of a certain subspace of the original Banach space. Using these solution operators we then get solutions to the equations:

$$
\begin{array}{ll}
d y / d t=A(t) y, & s \leqslant t, \quad y(s)=f, \\
d y / d s=-A(s) y, & s \leqslant t, \quad y(t)=f,
\end{array}
$$

when the initial condition $f$ is in the right subspace. Equation (5) is referred to as the forward equation and (6) is referred to as the backward equation.

Notice that for each $\omega \in \Omega$ we can consider an evolution equation of the same form as (2) by letting $A(t)=V(x(t, \omega))$. This brings us to our next proposition.

Proposition 4.4. If (A-1)-(A-3) hold, then for each $\omega \in \Omega$ there is a unique family of solution operators $M(s, t, \omega), 0 \leqslant s \leqslant t$, so that $y(s, \omega)=$ $M(s, t, \omega) \alpha$ satisfies

$$
d y / d s=-V(x(s, \omega)) y(s, \omega), \quad y(t, \omega)=\alpha,
$$

for each $\alpha \in D$. Moreover, if $\varphi: \Omega \rightarrow D \subseteq L$ is strongly measurable, then the map $\omega \rightarrow M(s, t, \omega) \varphi(\omega)$ is strongly measurable.

Proof. The hypotheses (A-1)-(A-3) and Lemma 4.3 guarantee that for each $\omega \in \Omega$ the operators $V(x(t, \omega))$ satisfy Goldstein's conditions. Here we use the continuity of paths of the process $X$ along with (A-4) to obtain the map $t$ $\rightarrow A(t) \alpha=V(x(t, \omega)) \alpha$ for $\alpha \in D$ is continuous, which is one of the conditions required by Goldstein. So for each $\omega \in \Omega$ Goldstein [4, Theorem. 1.1] guarantees the existence and uniqueness of a family of solution operators $\{M(s, t, \omega)$, $0 \leqslant s \leqslant t\}$ which satisfy equations (3) and (4). Then from (4) we obtain (7).

We give a brief outline of Goldstein's construction of the operators $M(s, t, \omega)$ because it is useful in proving the last statement of the proposition.

Let $a<b$. The construction of $M(a, b, \omega)$ is as follows: 
Let $\Delta=\left\{a=t_{0}<t_{1}<\cdots<t_{n}=b\right\}$ be a partition of the interval $[a, b]$ and, for $1 \leqslant j \leqslant n$, let $\tau_{j} \in\left[t_{j-1}, t_{j}\right]$. Define

$$
R_{\Delta}(\omega)=T_{x\left(\tau_{1}, \omega\right)}\left(t_{1}-a\right) T_{x\left(\tau_{2}, \omega\right)}\left(t_{2}-t_{1}\right) \cdots T_{x\left(\tau_{n}, \omega\right)}\left(b-t_{n-1}\right) .
$$

Goldstein has shown that if we define $|\Delta|=\max \left\{\left|t_{j}-t_{j-1}\right|: 1 \leqslant j \leqslant n\right\}$, then $R_{\Delta}(\omega)$ converges strongly to the solution operator $M(a, b, \omega)$ as $|\Delta| \rightarrow 0$. We now prove the last statement in the proposition.

Since $x(t): \Omega \rightarrow E$ is $\Sigma$-measurable for each $t \geqslant 0$, there are simple functions $y_{n}(t)$ mapping $\Omega$ into $E$ so that $y_{n}(t, \omega) \rightarrow x(t, \omega)$ for each $\omega \in \Omega$. The map $\omega \rightarrow T_{y_{n}\left(\tau_{i}, \omega\right)}\left(t_{i}-t_{i-1}\right)$ is a simple function from $\Omega$ to $B(L, L)$, the bounded linear operators on $L$, and, hence, so is the map $y_{m}: \Omega \rightarrow B(L, L)$ defined by

$$
y_{m}(\omega)=T_{y_{m}\left(\tau_{1}, \omega\right)}\left(t_{1}-a\right) \cdots T_{y_{m}\left(\tau_{n}, \omega\right)}\left(b-t_{n-1}\right) .
$$

Notice that by Lemmas 4.1, 4.3 and hypothesis (A-3), we obtain $y_{m}(\omega) \rightarrow$ $R_{\Delta}(\omega)$ strongly for each $\omega \in \Omega$. Now since $\varphi: \Omega \rightarrow L$ is strongly measurable, there are simple functions $\varphi_{n}: \Omega \rightarrow L$ so that $\varphi_{n}(\omega) \rightarrow \varphi(\omega)$ for each $\omega \in \Omega$ $[11$, p. 8].

Consider the map $z_{m}: \Omega \rightarrow L$ defined by $z_{m}(\omega)=y_{m}(\omega) \varphi_{m}(\omega)$. For each $m, z_{m}$ is a simple function and $z_{m}(\omega) \rightarrow R_{\Delta}(\omega) \varphi(\omega)$ for each $\omega \in \Omega$. Hence, the map $\omega \rightarrow R_{\Delta}(\omega) \varphi(\omega)$ is strongly measurable.

To complete the proof, choose a sequence of partitions $\left\{\Delta_{n}\right\}$ so that $\left|\Delta_{n}\right| \rightarrow 0$. Then

$$
M(a, b, \omega) \varphi(\omega)=\operatorname{Lim}_{n} R_{\Delta_{n}}(\omega) \varphi(\omega) .
$$

So by Lemma 4.2 , the map $\omega \rightarrow M(a, b, \omega) \varphi(\omega)$ is strongly measurable. This completes the proof.

It should be noted that assumption (A-2) can be weakened, but it is convenient to make the assumption later on and it will hold for the example we wish to consider.

Observe that we have shown that the map $\omega \rightarrow M(a, b, \omega) \varphi(\omega)$ is strongly measurable relative to the $\sigma$-field $\Sigma$ but we have the following corollary.

COROLLARY 4.5. If $\varphi: \Omega \rightarrow L$ is strongly measurable relative to $\Sigma_{b}$, then the map $\omega \rightarrow M(a, b, \omega) \varphi(\omega)$ is strongly measurable relative to $\Sigma_{b}$.

PROOF. Each $R_{\Delta_{n}}$ was the limit of simple functions which were $\Sigma_{b}$ measurable since they depended only on $x(t)$ for $t \leqslant b$.

COROLlARY 4.6. The strong conditional expectation of $M(a, b, \omega) \varphi(\omega)$ exists and 


$$
E_{x}\left(M(a, b) \varphi \mid \Sigma_{b}\right)=M(a, b) E_{x}\left(\varphi \mid \Sigma_{b}\right) .
$$

Proof. The proof of the existence is due to Scalora [14]. The proof of (8) follows exactly the proof of the real valued case and can be found in Griego and Hersh [6, Lemma 2].

There are several properties of the solution operators $M(s, t, \omega)$ which are shown in [4]. Since we shall use them later we list them here.

C.OROLlary 4.7. (a) $\|M(s, t, \omega)\| \leqslant 1$ for every $\omega \in \Omega$, and $0 \leqslant s \leqslant t$.

(b) $M(s, t, \omega) V(x(t, \omega))=V(x(t, \omega)) M(s, t, \omega)$.

(c) $M(s, r, \omega) M(t, s, \omega)=M(t, r, \omega)$ for each $\omega \in \Omega$ and $0 \leqslant t \leqslant s \leqslant r$.

Proof. The only thing not shown by Goldstein is (a), but it is obvious because each operator $T_{x}(t)$ is a contraction map.

We shall use the solution operators to define what we call a random evolution, which we will then use to define the expectation semigroup.

Definition 4.8. If $M(s, t, \omega), 0 \leqslant s \leqslant t$, is the family of solution operators for equation (7), define $M(t, \omega)=M(0, t, \omega)$ for each $\omega \in \Omega, t \geqslant 0$. The family $\{M(t), t \geqslant 0\}$ is called a random evolution.

LEMMA 4.9. If $0 \leqslant t \leqslant s \leqslant r$, and if $\alpha \in L$, then

$$
M(s, r, \omega) M(t, s, \omega) \alpha=M(t, s, \omega) M(s, r, \omega) \alpha
$$

for every $\omega \in \Omega$.

Proof. By Corollary 4.7(c) we have for each $\omega$, and $\alpha \in D, M(t, r, \omega) \alpha$ $=M(s, r, \omega) M(t, s, \omega) \alpha$. Fix $s$ so that $t \leqslant s \leqslant r$ and define $U(t, r, \omega)$ by

$$
U(t, r, \omega)=M(t, s, \omega) M(s, r, \omega) \text { for each } \omega \in \Omega \text {, and } t \leqslant s .
$$

Let $y(t, \omega)=U(t, r, \omega) \alpha$. Differentiating $y$ with respect to $t$, we obtain

$$
\begin{aligned}
\frac{d y}{d t} & =\frac{\partial}{\partial t} U(t, r, \omega) \alpha=\frac{\partial}{\partial t} M(t, s, \omega) M(s, r, \omega) \alpha \\
& =-V(x(t, \omega)) M(t, s, \omega) M(s, r, \omega) \alpha \\
& =-V(x(t, \omega)) y, \quad y(r, \omega)=\alpha .
\end{aligned}
$$

But we know $y_{1}(t, \omega)=M(t, r, \omega) \alpha$ is the unique solution to (7). Hence, $y_{1}=$ $y$, which is the desired result for $\alpha \in D$. Now since $M(s, r, \omega)$ and $M(t, s, \omega)$ are bounded operators and $D$ is dense in $L$, the result holds for all $\alpha \in L$.

Proposition 4.10. If $s, t \geqslant 0$, and if $\alpha \in D$, then $M(t+s, \omega) \alpha=M(s, \omega)$ $\cdot M\left(t, \theta_{s} \omega\right) \alpha$ for each $\omega \in \Omega$, where $\theta_{s}$ is the shift operator for the process $X$. 
Proof. Recall that by Definition 4.8, we have

$$
\begin{aligned}
M(t+s, \omega) \alpha & =M(0, t+s, \omega) \alpha, \\
M(s, \omega) \alpha & =M(0, s, \omega) \alpha, \\
M\left(t, \theta_{s} \omega\right) \alpha & =M\left(0, t, \theta_{s} \omega\right) \alpha .
\end{aligned}
$$

Now observe that $y_{1}(t, \omega)=M(s, t+s, \omega)$ is the unique solution of

$$
d y / d t=V\left(x\left(t, \theta_{s} \omega\right)\right) y=V(x(t+s, \omega)) y, \quad y(0)=\alpha .
$$

But, $y_{2}(t, \omega)=M\left(0, t, \theta_{s} \omega\right) \alpha$ also satisfies (12). Hence, $y_{1}=y_{2}$, or $M(s, t+s, \omega) \alpha=M\left(0, t, \theta_{s} \omega\right) \alpha$.

So, we now have

$$
M(0, s, \omega) M\left(0, t, \theta_{s} \omega\right) \alpha=M(0, s, \omega) M(s, t+s, \omega) \alpha,
$$

and, hence, equations (9), (10) and (11), along with Lemma 4.9 and Corollary 4.7, give us

$$
\begin{aligned}
M(s, \omega) M\left(t, \theta_{s} \omega\right) \alpha & =M(0, s, \omega) M(s, t+s, \omega) \alpha \\
& =M(0, t+s, \omega) \alpha=M(t+s, \omega) \alpha .
\end{aligned}
$$

This is the desired result.

Lemma 4.11. If $x, y \in E, s \geqslant 0$ and $\alpha \in D$ then

$$
V(x) T_{y}(s) \alpha=T_{y}(s) V(x) \alpha .
$$

Proof. Iet $x, y \in E, s \geqslant 0$ and $\alpha \in D$; then,

$$
\begin{aligned}
V(x) T_{y}(s) \alpha & =\lim _{t \rightarrow 0} \frac{T_{x}(t) T_{y}(s) \alpha-T_{y}(s) \alpha}{t}=\lim _{t \rightarrow 0} \frac{T_{y}(s) T_{x}(t) \alpha-T_{y}(s) \alpha}{t} \\
& =T_{y}(s) \lim _{t \rightarrow 0} \frac{T_{x}(t) \alpha-\alpha}{t}=T_{y}(s) V(x) \alpha .
\end{aligned}
$$

Corollary 4.12. If $x \in E, t, h \geqslant 0, \omega \in \Omega$ and $\alpha \in D$, then

$$
V(x) M(t, t+h, \omega) \alpha=M(t, t+h, \omega) V(x) \alpha .
$$

ProOF. This follows immediately from Lemma 4.11 and the construction of $M(t, t+h, \omega)$.

We are now ready to define the expectation semigroup.

DEFINITION 4.13. Let $f \in L^{p}(E, L, m), 1<p<\infty$; then define $(\widetilde{T}(t) f)(x)$ $=E_{x}(M(t) f(x(t)))$ for each $x \in E$, where the integral on the right exists as a Bochner integral. 
Notice that since $\|M(t)\| \leqslant 1$, by the results in $\S 3$ the integral will make sense for $m$-almost every $x$.

THEOREM 4.14. The operator $\widetilde{T}(t)$ is a bounded linear operator from $L^{P}(E, L, m)$ to itself for $1<p<\infty$.

Proof. The proof that $\widetilde{T}(t) f$ is measurable is exactly the same as in the proof of Theorem 3.6. Clearly, $\widetilde{T}(t)$ is linear.

We now show $\|\widetilde{T}(t) f\|_{p}<\infty$, if $f \in L^{p}(E, L, m)$. We have

$$
\begin{aligned}
\|\widetilde{T}(t) f\|_{p}^{p} & =\int_{E}\|\widetilde{T}(t) f(x)\|^{p} d m(x)=\int_{E}\left\|E_{x}(M(t) f(x(t)))\right\|^{p} d m(x) \\
& \leqslant \int_{E}\left\{E_{x}(\|M(t) f(x(t))\|)\right\}^{p} d m(x) \leqslant \int_{E}\left\{E_{x}\left(\|f(x(t))\|^{p}\right\}^{p} d m(x)\right. \\
& \leqslant \int_{E} E_{x}\left(\|f(x(t))\|^{p}\right) d m(x)=\int_{E}\|f(x)\|^{p} m P(t, d x) \\
& \leqslant \int_{E}\|f(x)\|^{p} d m(x)=\|f\|_{p}^{p}<\infty .
\end{aligned}
$$

Hence, $\widetilde{T}(t) f \in L^{P}(E, L, m)$. This completes the proof.

The above shows $\|\tilde{T}(t)\| \leqslant 1$. This proof is given for completeness. It can be found in more generality in Griego [5].

We now prove the main theorem of this section.

THEOREM 4.15. The family $\{\tilde{T}(t), t \geqslant 0\}$ is a strongly continuous contraction semigroup of operators on $L^{p}(E, L, m), 1<p<\infty$.

Proof. The strong continuity follows from Theorem 3.9. We have already shown that $\widetilde{T}(t)$ is a contraction map for each $t \geqslant 0$. It remains to show the semigroup property.

We have

$$
\begin{aligned}
\tilde{T}(t+s) f(x) & =E_{x}(M(t+s) f(x(t+s))) \\
& =E_{x}\left(M(s) M(t) \circ \theta_{s} f\left(x(t) \circ \theta_{s}\right)\right) \quad \text { (by Proposition 4.9) } \\
& =E_{x}\left(E_{x}\left(M(s) M(t) \circ \theta_{s} f\left(x(t) \circ \theta_{s}\right) \mid \Sigma_{s}\right)\right) \\
& =E_{x}\left(M(s) E_{x}\left(M(t) \circ \theta_{s} f\left(x(t) \circ \theta_{s}\right) \mid \Sigma_{s}\right)\right) \quad \text { (by Corollary 4.4) } \\
& =E_{x}\left(M(s) E_{x(s)}(M(t) f(x(t)))\right) \\
& =E_{x}(M(s) \widetilde{T}(t) f(x(s)))=\widetilde{T}(s) \widetilde{T}(t) f(x) .
\end{aligned}
$$

Further, $\tilde{T}(0) f(x)=E_{x}(M(0) f(x(0)))=f(x)$. Hence, $\{\tilde{T}(t), t \geqslant 0\}$ is a semigroup, which is the desired result. 
5. The operator-valued Feynman-Kac formula. In this section we shall use the semigroups defined in $\S \S 3$ and 4 to get the operator-valued FeynmanKac formula for a certain subspace of initial conditions contained in $L^{p}(E, L, m)$, where $1<p<\infty$. Since the results of $\S \S 3$ and 4 are going to be used, we shall assume everything we need to apply them, e.g. the excessivity of $m$ relative to the family $P(t, \cdot \cdot)$, the fact that $L$ is separable and reflexive, and assumptions (A-1) through (A-3).

Before we proceed to the derivation of the Feynman-Kac formula we need to define one more operator on a subspace of $L^{P}(E, L, m)$.

Definition 5.1. Recall that $D$ is the common domain of all the operators $V(x)$ on $L$. Define the operator $B$ by

$$
\begin{aligned}
O(B)=\left\{f \in L^{p}(E, L, m)\right. & f(x) \in D \text { a.e.m, } \\
& \text { and if } \left.F(x)=V(x) f(x), \text { then } F \in L^{p}(E, L, m)\right\}
\end{aligned}
$$

and

$$
B: D(B) \rightarrow L^{p}(E, L, m) \text { by } B f(x)=V(x) f(x) .
$$

LEMMA 5.2. The operator $B$ is closed and linear.

Proof. Clearly, $B$ is a linear operator, so it suffices to show $B$ is closed. To that end, assume $f_{n} \rightarrow f$ in $L^{p}(E, L, m)$, where $f_{n} \in D(B)$ for each $n$. Assume further that $B f_{n} \rightarrow g$ in $L^{P}(E, L, m)$. We need to show that $f \in D(B)$ and that $B f=g$.

Since $f_{n} \rightarrow f$ in $L^{p}(E, L, m)$, there is a subsequence $\left\{f_{n_{k}}\right\} \subseteq\left\{f_{n}\right\}$ so that $f_{n_{k}}(x) \rightarrow f(x)$ in $L$ for $m$-almost every $x$. Since $B f_{n} \rightarrow g$ in $L^{p}(E, L, m)$, we must have $B f_{n_{k}} \rightarrow g$ in $L^{p}(E, L, m)$ and, hence, there is a further subsequence $\left\{f_{n_{k_{i}}}\right\} \subseteq\left\{f_{n_{k}}\right\}^{k}$ so that:

$$
\text { (a) } f_{n_{k_{i}}}(x) \rightarrow f(x) \quad \text { a.e.m, and (b) } B f_{n_{k_{i}}}(x) \rightarrow g(x) \text { a.e.m. }
$$

But since each $V(x)$ is a closed operator we have $f(x) \in D(V(x))$ a.e. $m$ and $B f(x)=g(x)$ a.e. $m$.

We are now ready to define the space to which we shall restrict ourselves.

Definition 5.3. Define the space $D^{\prime}$ as follows:

$$
D^{\prime}=\left\{f \in O(B) \mid E_{x}(\|V(x(s)) f(x(t))\|) \leqslant F(s, t, f, x),\right.
$$

where

$$
\int_{0}^{\infty} \int_{0}^{t} e^{-\lambda t} F(s, t, f, x) d s d t<\infty \text { for all } \lambda>0
$$

and 


$$
\int_{E}|F(s, t, f, x)|^{p} d m(x)<\infty \quad \text { for } 0 \leqslant s \leqslant t<\infty .
$$

Let

$$
B C(E, L)=\left\{f: E \rightarrow L \mid\|f\|_{\infty}=\sup _{x \in E}\|f(x)\|<\infty \text { and } f \text { is continuous }\right\} .
$$

Finally, define

$$
D^{\prime \prime}=D^{\prime} \cap B C(E, L) .
$$

Before we proceed to deriving the Feynman-Kac formula, we make the following assumptions. Throughout the rest of this section assume:

(A-4) The map $x \rightarrow P_{x}$ is weakly continuous, i.e. if $Y: \Omega \rightarrow L$ is norm bounded and continuous and if $x_{n} \rightarrow x$, then $E_{x_{n}}(Y) \rightarrow E_{x}(Y)$.

(A-5) The map $(x, y) \rightarrow V(x) f(y)$ is jointly measurable for each $f \in D^{\prime \prime}$.

The approach we shall take is to restrict the semigroups of $\S \S 3$ and 4 to the Banach space $\overline{D^{\prime \prime}} \subseteq L^{p}(E, L, m)$. This will enable us to obtain a relationship between the resolvent operators of the semigroups, which will in turn allow us to derive the Feynman-Kac formula. To do this we must first show that under certain conditions $\bar{T}(h)$ and $\widetilde{T}(h)$ leave $D^{\prime \prime}$ invariant for all $h \geqslant 0$.

Proposition 5.4. If $f \in D^{\prime \prime}$ and $h \geqslant 0$, then $\bar{T}(h) f \in D^{\prime \prime}$.

Proof. We first show $\bar{T}(h) f \in B C(E, L)$. Since $f \in B C(E, L)$, we know $f(x(h)): \Omega \rightarrow L$ is bounded and it is clearly continuous. So, if $x_{n} \rightarrow x$ in $E$, then by (A-4) $E_{x_{n}}(f(x(h))) \rightarrow E_{x}(f(x(h)))$, which gives us the continuity of $\bar{T}(h) f$. It is clearly bounded by $\|f\|_{\infty}$.

We must now show that $\bar{T}(h) f \in D(B)$. To that end, let $x \in E$. Then we have

$$
E_{x}(\|V(x) f(x(h))\|)=E_{x}(\|V(x(0)) f(x(h))\|) \leqslant F(0, h, f, x)<\infty \quad \text { a.e.m. }
$$

The reason for the second to last inequality is that $f \in D^{\prime}$. Now, since $V(x)$ is a closed operator, we get

$$
\bar{T}(h) f(x)=E_{x}(f(x(h))) \in \mathcal{D}(V(x))
$$

and further:

$$
V(x) \bar{T}(h) f(x)=E_{x}(V(x) f(x(h))) .
$$

The fact that $x \rightarrow V(x) \bar{T}(h) f(x)$ is in $L^{p}(E, L, m)$ now follows from the fact that $F(0, h, f, \cdot) \in L^{p}(E, L, m)$.

Finally we need to show that there is a function $\bar{F}(s, t, \bar{T}(h) f, x)$ satisfying (*) and (**). Notice that by an argument similar to the one which gave us (13) we obtain 


$$
V(x(s)) \bar{T}(h) f(x(t))=E_{x(t)}(V(x(s)) f(x(h))),
$$

and hence,

$$
\begin{aligned}
\|V(x(s)) \bar{T}(h) f(x(t))\| & \leqslant E_{x(t)}(\|V(x(s)) f(x(h))\|) \\
& =E_{x}\left(\|V(x(s)) f(x(t+h))\| \mid \Sigma_{t}\right) .
\end{aligned}
$$

Now taking expectations we get

$$
E_{x}(\|V(x(s)) \bar{T}(h) f(x(t))\|) \leqslant E_{x}(\|V(x(s)) f(x(t+h))\|) \leqslant F(s, t+h, f, x) .
$$

Letting $\bar{F}(s, t, \bar{T}(h) f, x)=F(s, t+h, f, x)$, we obtain the desired result. This completes the proof.

Proposition 5.5. If $f \in D^{\prime \prime}$ and $h \geqslant 0$, then $\widetilde{T}(h) f \in D^{\prime \prime}$.

Proof. That $\widetilde{T}(h) f \in B C(E, L)$ follows exactly as in the proof that $\bar{T}(h) f \in$ $B C(E, L)$ (Proposition 5.4) and the fact that $\|M(h)\| \leqslant 1$.

We now show $\widetilde{T}(h) f \in D(B)$. Using the same argument as in Proposition 5.4, it suffices to show:

(a) $E_{x}(\|V(x) M(h) f(x(h))\|)<\infty$ a.e. $m$ and

(b) $\int_{E}\left\|E_{x}(V(x) M(h) f(x(h)))\right\| d m(x)<\infty$.

But $E_{x}(\|V(x) M(h) f(x(h))\|) \leqslant E_{x}(\|V(x) f(x(h))\|)$ by Corollary 4.12 , and since $E_{x}(\|V(x) f(x(h))\|) \leqslant F(0, h, f, x)$, both (a) and (b) follow.

We now need to show that $\widetilde{T}(h) f$ satisfies the defining inequality for $D^{\prime}$. Using Corollary 4.12 we obtain

$$
\begin{aligned}
E_{x}(\| V(x(s)) & \widetilde{T}(h) f(x(t)) \|)=E_{x}\left(\left\|V(x(s)) E_{x(t)}(M(h) f(x(h)))\right\|\right) \\
& =E_{x}\left(\left\|E_{x(t)}(V(x(s)) M(h) f(x(h)))\right\|\right) \leqslant E_{x}\left(E_{x(t)}(\|V(x(s)) f(x(h))\|)\right) \\
& =E_{x}\left(E_{x}\left(\|V(x(s)) f(x(t+h))\| \mid \Sigma_{t}\right)\right)=E_{x}(\|V(x(s)) f(x(t+h))\|) \\
& \leqslant F(s, t+h, f, x) .
\end{aligned}
$$

Letting $\widetilde{F}(s, t, \widetilde{T}(h) f, x)=F(s, t+h, f, x)$ we obtain the desired result.

COROLlaRY 5.6. The operators $\widetilde{T}(h), \bar{T}(h)$ leave $D^{\prime \prime}$ invariant for all $h \geqslant 0$.

We now assume that the hypotheses of Proposition 5.5 hold throughout the rest of this section. Since $\bar{T}(h)$ and $\widetilde{T}(h)$ leave $D^{\prime \prime}$ invariant for every $h \geqslant 0$, we 
can consider $\{\bar{T}(h), h \geqslant 0\}$ and $\{\widetilde{T}(h), h \geqslant 0\}$ as strongly continuous contraction semigroups on the Banach space $\overline{D^{\prime \prime}}$. Let $\bar{A}$ and $\widetilde{A}$, respectively, denote their infinitesimal generators, and let $\bar{R}_{\lambda}$ and $\widetilde{R}_{\lambda}$ denote their respective resolvents. We now proceed to show $u(t, x)=\tilde{T}(t) f(x)$, which is the operator-valued Feynman-Kac formula, satisfies (1) when $f$ is in a certain subspace of $\bar{D}^{\prime \prime}$.

LEMMA 5.7. If $f \in D^{\prime \prime}$, then

$$
\int_{0}^{\infty} \int_{0}^{t} E_{x}\left(e^{-\lambda t}\|M(s) V(x(s)) f(x(t))\|\right) d s d t<\infty
$$

for every $\lambda>0$.

Proof. This follows from the fact that $f \in D^{\prime}$ and that $F(s, t, f, x)$ satisfies (*) and $\|M(s)\| \leqslant 1$ for all $s$.

COROLlaRY 5.8. If $f \in D^{\prime \prime}$, then the order of integration in

$$
\int_{0}^{\infty} \int_{0}^{t} E_{x}\left(e^{-\lambda t} M(s) V(x(s)) f(x(t))\right) d s d t
$$

is immaterial.

Proof. Apply the Fubini-Tonelli Theorem. Notice here we use (A-5) to get joint measurability of $M(s) V(x(s)) f(x(t))$.

LEMMA 5.9. If $f \in D^{\prime \prime}$, then

$$
\widetilde{R}_{\lambda} f-\bar{R}_{\lambda} f=\bar{R}_{\lambda} B \widetilde{R}_{\lambda} f \text { for all } \lambda>0
$$

Proof. We have

$$
\begin{aligned}
\widetilde{R}_{\lambda} f(x)-\bar{R}_{\lambda} f(x) & =\int_{0}^{\infty} e^{-\lambda t}(\widetilde{T}(t) f-\bar{T}(t) f)(x) d t \\
& =\int_{0}^{\infty} e^{-\lambda t} E_{x}((M(t)-I) f(x(t))) d t
\end{aligned}
$$

But recall that Proposition 4.4 gives us

$$
-(d / d s) M(s, t) f(x(t))=V(x(s)) M(s, t) f(x(t)) .
$$

So upon integration, we obtain

$$
\int_{0}^{t} V(x(s)) M(s, t) f(x(t)) d s=-\left.M(s, t) f(x(t))\right|_{s=0} ^{s=t}=(M(t)-I) f(x(t))
$$

Returning to our original equation, we get 


$$
\begin{aligned}
& \widetilde{R}_{\lambda} f(x)-\bar{R}_{\lambda} f(x)=\int_{0}^{\infty} e^{-\lambda t} E_{x}\left(\int_{0}^{t} V(x(s)) M(s, t) f(x(t)) d s\right) d t \\
& =\int_{0}^{\infty} \int_{0}^{t} E_{x}\left(e^{-\lambda t} V(x(s)) M(s, t) f(x(t))\right) d s d t \quad \text { (by Corollary 5.8) } \\
& =\int_{0}^{\infty} \int_{s}^{\infty} E_{x}\left(e^{-\lambda t} V(x(s)) M(s, t) f(x(t))\right) d t d s \quad \text { (by Corollary 5.8) } \\
& =\int_{0}^{\infty} \int_{0}^{\infty} E_{x}\left(e^{-\lambda(t+s)} V(x(s)) M(s, t+s) f(x(t+s))\right) d t d s \\
& =\int_{0}^{\infty} e^{-\lambda s} \int_{0}^{\infty} e^{-\lambda t} E_{x}\left(E_{x}\left(V(x(s)) M(s, t+s) f(x(t+s)) \mid \Sigma_{s}\right)\right) d t d s \\
& =\int_{0}^{\infty} e^{-\lambda s} \int_{0}^{\infty} e^{-\lambda t} E_{x}\left(V(x(s)) E_{x}\left(M(s, t+s) f(x(t+s)) \mid \Sigma_{s}\right)\right) d t d s
\end{aligned}
$$

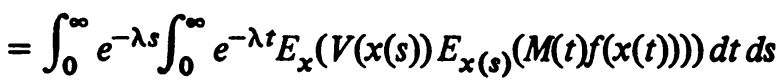

$$
\begin{aligned}
& =\int_{0}^{\infty} e^{-\lambda s} \int_{0}^{\infty} e^{-\lambda t} E_{x}(V(x(s)) \widetilde{T}(t) f(x(s))) d t d s \\
& =\int_{0}^{\infty} e^{-\lambda s} \int_{0}^{\infty} e^{-\lambda t} E_{x}(B \widetilde{T}(t) f(x(s))) d t d s \\
& =\int_{0}^{\infty} e^{-\lambda s} \int_{0}^{\infty} e^{-\lambda t} \bar{T}(s) B \widetilde{T}(t) f(x) d t d s \\
& =\int_{0}^{\infty} e^{-\lambda s} \bar{T}(s) B\left(\int_{0}^{\infty} e^{-\lambda t} \widetilde{T}(t) f d t\right)(x) d s
\end{aligned}
$$

(by Theorem 3.6 and Lemma 5.2)

$$
=\int_{0}^{\infty} e^{-\lambda s} \bar{T}(s) B \widetilde{R}_{\lambda} f(x) d s=\bar{R}_{\lambda} B \widetilde{R}_{\lambda} f(x) .
$$

This completes the proof.

THEOREM 5.10. The operator $\widetilde{R}_{\lambda}$ maps $D^{\prime \prime}$ into $D(\bar{A})$. Further, if $f \in$ $\widetilde{R}_{\lambda}\left(D^{\prime \prime}\right)$, then $\widetilde{A} f=\bar{A} f+B f$.

Proof. If $f \in D^{\prime \prime}$, then by Lemma 5.9 we have $\widetilde{R}_{\lambda} f=\bar{R}_{\lambda}\left(B \widetilde{R}_{\lambda}-I\right) f \in$ $\bar{R}_{\lambda}\left(\bar{D}^{\prime \prime}\right)=$ range of $\bar{R}_{\lambda}=O(\bar{A})$. This establishes the first statement.

Now if $f \in \widetilde{R}_{\lambda}\left(D^{\prime \prime}\right)$, then there is a $g \in D^{\prime \prime}$ so that $f=\widetilde{R}_{\lambda} g$. Then by Lemma 5.9 , we have

which implies

$$
\widetilde{R}_{\lambda} g-\bar{R}_{\lambda} g=\bar{R}_{\lambda} B \widetilde{R}_{\lambda} g
$$

$$
\widetilde{R}_{\lambda}^{-} g=\bar{R}_{\lambda} g+\bar{R}_{\lambda} B f
$$

hence,

$$
f=\bar{R}_{\lambda}(g+B f) \text {. }
$$

Now apply $\lambda-\bar{A}$ to both sides of the above to obtain

$$
\lambda f-\bar{A} f=g+B f=(\lambda-\widetilde{A}) f+B f=\lambda f-\tilde{A} f+B f .
$$

Rearranging terms, we obtain 


$$
\widetilde{A} f=\bar{A} f+B f \text {. }
$$

This completes the proof.

We are now ready to state and prove our main result.

THEOREM 5.11. If $D_{0}=\left\{f \in D^{\prime \prime} \cap D(\widetilde{A})\left[\widetilde{A} f \in D^{\prime \prime}\right\}\right.$, and if $f \in D_{0}$, then the initial value problem $\partial u / \partial t=\bar{A} u+B u, u(0)=f$, has a solution given by $u(t)=\widetilde{T}(t) f$

Proof. It is a standard result in the theory of semigroups $[15, \mathrm{p} .239]$ that if $f \in D(\widetilde{A})$, then $u(t)$ as defined above satisfies $\partial u / \partial t=\widetilde{A} u$. Hence, by Theorem 5.10 it suffices to show that $u(t) \in \widetilde{R}_{\lambda}\left(D^{\prime \prime}\right)$ for every $t \geqslant 0$.

Recall that $f \in D_{0} \subseteq D(\widetilde{A})$; hence, $u(t)=\widetilde{T}(t) f \in O(\widetilde{A})[15$, p. 239]. Let $v(t)=(\lambda-\widetilde{A}) u(t)$. Then $v(t)=(\lambda-\widetilde{A}) \widetilde{T}(t) f=\widetilde{T}(t)(\lambda-\widetilde{A}) f$. But, since $f \in$ $D_{0}$, we have that both $f$ and $\widetilde{A} f$ belong to $D^{\prime \prime}$. Therefore, $(\lambda-\widetilde{A}) f \in D^{\prime \prime}$, and by Corollary 5.6, we have $v(t)=\widetilde{T}(t)(\lambda-\widetilde{A}) f \in D^{\prime \prime}$. Furthermore, $\widetilde{R}_{\lambda} v(t)=u(t)$, hence $u(t) \in \widetilde{R}_{\lambda}\left(D^{\prime \prime}\right)$ for each $t \geqslant 0$. This completes the proof.

We now proceed to show that $D_{0}$ is nonempty and, in fact, under the proper conditions is dense in $\overline{D^{\prime \prime}}$.

LEMMA 5.12. If $f \in D^{\prime \prime}$ and if we define the function $g$ by $g(K)=$ $\int_{0}^{K} \widetilde{T}(t) f d t$, and if $\int_{0}^{K} F(s, t+h, f, x) d h$ satisfies $(*)$ and $(* *)$, then $g(K) \in D^{\prime \prime}$.

Proof. Observe that $g(K): E \rightarrow L$ is bounded by $K\|f\|_{\infty}$. We now show that it is continuous. Let $x_{n} \rightarrow x$ in $E$. Then

$$
g(K)\left(x_{n}\right)=\int_{0}^{K} E_{x_{n}}(M(t) f(x(t))) d t=E_{x_{n}}\left(\int_{0}^{K} M(t) f(x(t)) d t\right)
$$

But,

$$
E_{x_{n}}\left(\int_{0}^{K} M(t) f(x(t)) d t\right) \rightarrow E_{x}\left(\int_{0}^{K} M(t) f(x(t)) d t\right)
$$

because of (A-4) and the fact that $\int_{0}^{K} M(t) f(x(t)) d t$ is a bounded function from $\Omega$ to $L$. But notice that

$$
E_{x}\left(\int_{0}^{K} M(t) f(x(t)) d t\right)=g(K)(x)
$$

Hence, $g(K)\left(x_{n}\right) \rightarrow g(K)(x)$, and $g(K)$ is continuous. Therefore, $g(K) \in$ $B C(E, L)$.

That $g(K) \in D^{\prime}$ follows directly from the fact that $\int_{0}^{K} F(s, t+h, f, x) d h$ satisfies $(*)$ and $(* *)$.

THEOREM 5.13. If the hypotheses of Lemma 5.12 are satisfied for $K=$ $1 / n$ for every $n$, and if $D^{\prime \prime} \neq\{0\}$, then $D_{0} \neq\{0\}$. 
Proof. Let $f \neq 0$ be in $D^{\prime \prime}$ and define $f_{n}=n \int_{0}^{1 / n} \widetilde{T}(t) f d t$.

Then by Lemma 5.12, $f \in \mathcal{D}^{\prime \prime}$. But, $f_{n} \in \mathcal{O}(\widetilde{A})$ and $\widetilde{A} f_{n}=n \widetilde{T}(1 / n) f-n f \in D^{\prime \prime}$. Hence, $f_{n} \in D_{0}$ for every $n$. Now, if $D_{0}=\{0\}$, then $f_{n}=0$ for every $n$. But, $f_{n} \rightarrow f$ and so if $D_{0}=\{0\}$, then $f=0$ which is a contradiction. Hence, $D_{0} \neq$ $\{0\}$ and the proof is complete.

CoRollary 5.14. If $M$ is a linear subspace of $D^{\prime \prime}$ which is dense in $\bar{D}^{\prime \prime}$, and if the hypotheses of Theorem 5.13 are satisfied by each $f \in M$, then $D_{0}$ is dense in $\overline{D^{\prime \prime}}$.

Proof. Let $f \in \bar{D}^{\prime \prime}$ and let $\epsilon>0$. Choose $g \in M$ so that $\|f-g\|<\epsilon / 2$. Let $g_{n}=n \int_{0}^{1 / n} \widetilde{T}(t) g d t$. Then for $n$ sufficiently large $\left\|g_{n}-g\right\|<\epsilon / 2$ and hence, $\left\|f-g_{n}\right\| \leqslant\|f-g\|+\left\|g_{n}-g\right\|<\epsilon$. But Theorem 5.13 guarantees that $g_{n} \in D_{0}$ for all $n$. This completes the proof.

Finally we give necessary and sufficient conditions on the operators $\bar{A}$ and $B$ so that $\bar{A}+B$ is actually an extension of the operator $\widetilde{A}$.

DEFINITION 5.15. We define $G$ to be the space of functions on which $\widetilde{A}$ $=\bar{A}+B$, i.e.

$$
G=\{f \in \mathcal{O}(\tilde{A}) \cap D(B) \cap D(A): \tilde{A} f=\bar{A} f+B f\} .
$$

THEOREM 5.16. If $D(\widetilde{A})=G$, then $\left.(\bar{A}+B)\right|_{G}$ is a closed operator and, conversely, if $\left.(\bar{A}+B)\right|_{G}$ is closed, then $D(\widetilde{A})=G$.

PROof. To show the first implication let $f_{n} \rightarrow f$ where $f_{n} \in D(\bar{A}+B) \cap$ $G$ and assume $(\bar{A}+B) f_{n} \rightarrow g$. Since each $f_{n} \in G$ we have first each $f_{n} \in D(\widetilde{A})$ and second that $\widetilde{A} f_{n}=(\bar{A}+B) f_{n} \rightarrow g$. Since $\widetilde{A}$ is a closed operator $f \in D(\widetilde{A})$ and $\widetilde{A} f=g$. But $f \in \mathcal{O}(\widetilde{A})$ implies $f \in G$ and hence $\widetilde{A} f=(\bar{A}+B) f=g$. Hence $\left.(\bar{A}+B)\right|_{G}$ is a closed operator.

To show the converse we notice that by Theorem 5.10, $\widetilde{R}_{\lambda}\left(D^{\prime \prime}\right) \subseteq G$ and clearly $G \subseteq D(\widetilde{A})=\widetilde{R}_{\lambda}\left(\overline{D^{\prime \prime}}\right)$. We need therefore only show that if $\left.(\bar{A}+B)\right|_{G}$ is closed then $\widetilde{R}_{\lambda}\left(\bar{D}^{\prime \prime}\right) \subseteq G$. In view of the above observation, this amounts to showing that if $f \in \widetilde{R}_{\lambda}\left(\overline{D^{\prime \prime}}\right)-\widetilde{R}_{\lambda}\left(\mathcal{D}^{\prime \prime}\right)$ then $f \in G$.

To that end let $f \in \widetilde{R}_{\lambda}\left(\overline{D^{\prime \prime}}\right)-\widetilde{R}_{\lambda}\left(D^{\prime \prime}\right)$. There is some element $g \in \overline{D^{\prime \prime}}-D^{\prime \prime}$ so that $\widetilde{R}_{\lambda} g=f$. Since $g \in{\overline{D^{\prime \prime}}}^{\prime \prime}$ there is a sequence $\left\{g_{n}\right\} \subseteq D^{\prime \prime}$ so that $g_{n} \rightarrow g$. Define $f_{n}=\widetilde{R}_{\lambda} g_{n}$. Now each $f_{n}$ is in $\widetilde{R}_{\lambda}\left(D^{\prime \prime}\right)$ and further,

$$
f_{n}=\widetilde{R}_{\lambda} g_{n} \rightarrow \widetilde{R}_{\lambda} g=f
$$

because $g_{n} \rightarrow g$ and $\widetilde{R}_{\lambda}$ is a bounded operator. We shall show that $\left\{(\bar{A}+B) f_{n}\right\}$ converges. Note that by Theorem $5.10,(\bar{A}+B) f_{n}=\widetilde{A} f_{n}$. Now $\left\{(\lambda-\widetilde{A}) f_{n}\right\}=$ $\left\{(\lambda-\widetilde{A}) \widetilde{R}_{\lambda} g_{n}\right\}=\left\{g_{n}\right\}$, which is a convergent sequence, and since the sequence $\left\{\lambda f_{n}\right\}$ converges by $(15)$ we must have that the sequence $\left\{\widetilde{A} f_{n}\right\}$ converges so by the 
above note the sequence $\left\{(\bar{A}+B) f_{n}\right\}$ converges to some element $h$, namely $h=$ $\lambda f-g$.

We now have first $f_{n} \rightarrow f$ and each $f_{n} \in G$ and second $(\bar{A}+B) f_{n} \rightarrow h$. Since we are assuming that $\left.(\bar{A}+B)\right|_{G}$ is a closed operator we can conclude that $f \in G$ and $(\bar{A}+B) f=h$. In particular, $f \in G$ which is the desired result.

COROLlARY 5.17. The operator $\bar{A}+B$ is an extension of $\widetilde{A}$ if and only if $\left.(\bar{A}+B)\right|_{G}$ is a closed operator.

Proof. This follows directly from Theorem 5.16.

6. Example. In this section we shall apply the above results to a specific example.

Let $X=\left(\Omega, \Sigma, \Sigma_{t}, x(t), P_{x}, x \in R^{1}\right)$ be a Brownian motion on the real line, and suppose $L=L^{2}(R)$. Recall the transition probabilities of Brownian motion are given by

$$
P(t, x, A)=\frac{1}{\sqrt{2 \pi t}} \int_{A} \exp \left[\frac{-(x-y)^{2}}{2 t} \cdot\right] d y .
$$

The measure $m$ in this case will be Lebesgue measure on $R^{1}$. It is easily seen that $m$ is excessive relative to the family $P(t, ;)$. Now for every $x \in R$ define $T_{x}(t): L \rightarrow L$ by $T_{x}(t) \varphi(\theta)=\varphi(\theta+g(x) t)$, where $g$ is continuous, bounded and $g(x) \neq 0$ for every $x \in R$ and further, for every $t>0$,

$$
\int_{-\infty}^{\infty}\left\{\frac{1}{\sqrt{2 \pi t}} \int_{-\infty}^{\infty} \lg (y) \mid \exp \left[\frac{-(x-y)^{2}}{2 t}\right] d y\right\}^{2} d x<\infty .
$$

Then it can be shown $\left[1\right.$, p. 43] that $T_{x}(t)$ is a strongly continuous contraction semigroup of operators on $L$ and the infinitesimal generator of $T_{x}(t)$ is given by

$$
D(V(x))=\left\{\varphi \in L^{2}(R) \mid \varphi \in A C_{\mathrm{loc}}(R) \text { and } \partial \varphi / \partial \theta \in L^{2}(R)\right\} .
$$

where $A C_{\text {loc }}(R)$ denotes the set of all locally absolutely continuous functions from $R$ to $R$ and

$$
V(x) \varphi=g(x) \partial \varphi / \partial \theta
$$

It is clear that (A-1) through (A-5) are satisfied by the above.

THEOREM 6.1. Under the conditions set out above, the initial value problem:

$$
\partial u / \partial t=1 / 2 \partial^{2} u / \partial x^{2}+g(x) \partial u / \partial \theta, \quad u(0)=f,
$$

has a solution if $f \in D_{0}$.

Proof. Theorem 5.11. 
We should note here that $D_{0} \neq\{0\}$. We shall show this by exhibiting an element $f \in D^{\prime \prime}-\{0\}$ which satisfies the hypotheses of Lemma 5.12 and Theorem 5.13. From this $f$ we can construct elements of $D_{0}$.

THEOREM 6.2. Let $f: R \rightarrow L^{2}(R)$ be defined by $f(x, \theta)=\exp \left[-(x-\theta)^{2}\right]$. Then $f \in D^{\prime \prime}$.

Proof. The function $f$ is clearly in $D(B)$ and is clearly bounded. We show that $f$ is continuous by observing that if $x_{n} \rightarrow x$, since $\left\|f\left(x_{n}\right)\right\|_{2}=\|f(x)\|_{2}$ for every $n$ and since $f\left(x_{n}, \theta\right) \rightarrow f(x, \theta)$ for every $\theta$, then $\left\|f\left(x_{n}\right)-f(x)\right\|_{2} \rightarrow 0$ $[13$, p. 73].

Now we need to show that $f$ satisfies the defining inequality for $D^{\prime \prime}$.

Notice

$$
\begin{aligned}
V(x(s)) f(x(t), \theta) & =g(x(s)) \frac{\partial \exp \left[-(x(t)-\theta)^{2}\right]}{\partial \theta} \\
& =2 g(x(s))(x(t)-\theta) \exp \left[-(x(t)-\theta)^{2}\right]
\end{aligned}
$$

So we can compute $\|V(x(s)) f(x(t))\|$ by

$$
\begin{aligned}
\|V(x(s)) f(x(t))\|^{2} & =\int_{-\infty}^{\infty} 4|g(x(s))|^{2}|x(t)-\theta|^{2} \exp \left[-2(x(t)-\theta)^{2}\right] d \theta \\
& =\left.4 \lg (x(s))\right|^{2} \int_{-\infty}^{\infty} u^{2} \exp \left(-2 u^{2}\right) d u .
\end{aligned}
$$

Now let $F(s, t, f, x)=K E_{x}(|g(x(s))|)$, where

$$
K^{2}=\int_{-\infty}^{\infty} 4 u^{2} \exp \left(-2 u^{2}\right) d u<\infty .
$$

We need to check that $F$ satisfies (*) and (**). We have

$$
\int_{0}^{\infty} \int_{0}^{t} e^{-\lambda t} F(s, t, f, x) d s d t \leqslant \int_{0}^{\infty} K\|g\|_{\infty} t e^{-\lambda t} d t<\infty
$$

hence, $F$ satisfies (*). To check (**) note that

$$
\begin{aligned}
\int_{-\infty}^{\infty}|F(s, t, f, x)|^{2} d x & =\int_{-\infty}^{\infty} K^{2}\left(E_{x}(|g(x(s))|)\right)^{2} d x \\
& =\int_{-\infty}^{\infty} \frac{K^{2}}{\sqrt{2 \pi s}}\left\{\int_{-\infty}^{\infty}|g(y)| \exp \left[\frac{-(x-y)^{2}}{2 s}\right] d y\right\}^{2} d x<\infty
\end{aligned}
$$

by the restrictions placed on $g$. Hence, $F$ satisfies $(* *)$. This completes the proof.

Now in order to show that $D_{0} \neq 0$, by Theorem 5.13 we need only show $f$ satisfies the hypotheses of Lemma 5.12 for $K=1 / n$, for all $n$.

THEOREM 6.3. If $f$ is defined as in Theorem 6.2 and if $F(s, t, f, x)$ is the function obtained in Theorem 6.2, then $\int_{0}^{1 / m} F(s, t+h, f, x) d h$ satisfies (*) and (**). 
Proof. Notice that $F(s, t+h, f, x)=K E_{x}(g(x(s)))$, where $K$ is defined in the proof of Theorem 6.2 so we have

$$
\int_{0}^{1 / m} F(s, t+h, f, x) d h=\frac{K}{m} E_{x}(g(x(s))) .
$$

This clearly satisfies (*) and (**) and the proof is complete.

Corollary 6.4. The subspace $D_{0}$ is nontrivial, i.e. $D_{0} \neq\{0\}$.

Proof. Theorems 5.13, 6.2 and 6.3.

COROLlary 6.5. The subspace $M$ is contained in $D_{0}$, where

$$
M=\left\{\sum_{i=1}^{n} g_{i}(x) h_{i}(\theta) \mid g_{i}, h_{i} \in C_{c}(R), n \in N, h \in D(V(x))\right\} .
$$

Proof. It is an easy computation to show that if $f(x, \theta)=r(x) h(\theta)$, then the function $F$ is given by $F(s, t, f, x)=K\|h\|_{\infty} E_{x}(|g(x(s))|)$. The corollary then follows as did Corollary 6.4.

7. Remarks. As was noted in the introduction, a number of people have considered the Feynman-Kac formula in studying probabilistic phenomena.

In [8] Helms constructs a stochastic process which corresponds to the classical Feynman-Kac formula. In that case the measure that Helms obtains is not necessarily a probability measure. The most one can say is that it is $\sigma$ finite. A question remains as to what type of stochastic process can be associated with the operator-valued Feynman-Kac formula, and of course what type of measure is associated with it. In [7] Griego and Moncayo construct the process in the discrete case.

Finally, the assumption that the semigroups $T_{x}(t)$ are mutually commutative is undesirable. The results of Hersh and Papanicolaou [9] do not depend on this commutativity. In attacking the problem from the point of view of resolvents, difficulties are encountered in showing the measurability of the multiplicative operator functional and in the computation of the resolvent equation obtained in Lemma 5.9. So the question remains open on sufficient conditions in the noncommutative case.

\section{REFERENCES}

1. P. L. Butzer and H. Berens, Semi-groups of operators and approximation, Die Grundlehren der math. Wissenschaften, Band 145, Springer-Verlag, New York, 1967. MR 37 \#5588.

2. N. Dunford and J. T. Schwartz, Linear operators. I: General theory, Pure and Appl. Math., vol. 7, Interscience, New York, 1958. MR 22 \#8302.

3. E. B. Dynkin, Markov processes, Fizmatgiz, Moscow, 1963; English transl., Vol. I, Die Grundlehren der math. Wissenschaften, Band 121, Academic Press, New York; SpringerVerlag, Berlin, 1965. MR 33 \#1886; \#1887. 
4. J. Goldstein, A bstract evolution equations, Trans. Amer. Math. Soc. 141 (1969), 159-185. MR 40 \#789.

5. R. Griego, Dual random evolutions, Univ. of New Mexico Tech. Report No. 301, September, 1974.

6. R. Griego and R. Hersh, Theory of random evolutions with applications to partial differential equations, Trans. Amer. Math. Soc. 156 (1971), 405-418. MR 43 \#1261.

7. R. Griego and A. Moncayo, Random evolutions and piecing out of Markov processes, Bol. Soc. Mat. Mexicana 15 (1970), 22-29.

8. L. L. Helms, Markov processes with creation of mass, Z. Wahrscheinlichkeitstheorie und Verw. Gebiete 7 (1967), 225-234. MR 36 \#3411.

9. R. Hersh and G. Papanicolaou, Non-commuting random evolutions, and an operatorvalued Feynman-Kac formula, Comm. Pure Appl. Math. 25 (1972), 337-367. MR 46 \#10038.

10. M. Kac, On some connections between probability theory and differential and integral equations, Proc. Second Berkeley Sympos. Math. Statist. and Probability (1950), Univ. of California Press, Berkeley, Calif., 1951, pp. 189-215. MR 13, 568.

11. S. Khalili, Bochner measurability, Univ. of Pittsburgh Research Report No. 73-04, 1973.

12. T. Leviatan, On Markov processes with random starting times, Ann. Probability 1 (1973), 223-231. MR 50 \#11473.

13. W. Rudin, Real and complex analysis, McGraw-Hill, New York, 1966. MR 35 \#1420.

14. F. S. Scalora, Abstract martingale convergence theorems, Pacific J. Math. 11 (1961), 347-374. MR 23 \#A684.

15. K. Yosida, Functional analysis, Die Grundlehren der math. Wissenschaften, Band 123, Academic Press, New York; Springer-Verlag, Berlin, 1965. MR 31 \#5054.

16. A. Zaanen, Integration, 2nd ed., Wiley, New York, 1967. MR 36 \#5286.

\section{DEPARTMENT OF MATHEMATICS, LOYOLA MARYMOUNT UNIVERSITY, LOS ANGELES, CALIFORNIA 90045}

